\title{
Missing GRB host galaxies in deep mid-infrared observations: implications on the use of GRBs as star formation tracers
} \author{
Lee Armus ${ }^{\S}$ and Daniel Devost \\ ${ }^{*}$ University of Arizona, Tucson, AZ 85721, USA \\ $\dagger$ University of Crete, GR-71003, Heraklion, Greece \\ ${ }^{* *}$ University of Rochester, Rochester, NY 14627, USA \\ ${ }^{\ddagger}$ European Southern Observatory, Santiago 19, Chile \\ ${ }^{\S}$ Spitzer Science Center, Pasadena, CA 91125, USA \\ ${ }^{\natural}$ Cornell University, Ithaca, NY 14853, USA
}

Emeric Le Floc'h*, Vassilis Charmandaris ${ }^{\dagger}$, Bill Forrest**, Félix Mirabel ${ }^{\ddagger}$,

\begin{abstract}
.
We report on the first mid-infrared observations of 16 GRB host galaxies performed with the Spitzer Space Telescope, and investigate the presence of evolved stellar populations and dustenshrouded star-forming activity associated with GRBs. Only a very small fraction of our sample is detected by Spitzer, which is not consistent with recent works suggesting the presence of a GRB host population dominated by massive and strongly-starbursting galaxies (SFR $\gtrsim 100 \mathrm{M}_{\odot} \mathrm{yr}^{-1}$ ). Should the GRB hosts be representative of star-forming galaxies at high redshift, models of galaxy evolution indicate that $\gtrsim 50 \%$ of GRB hosts would be easily detected at the depth of our midinfrared observations. Unless our sample suffers from a strong observational bias which remains to be understood, we infer in this context that the GRBs identified with the current techniques can not be directly used as unbiased probes of the global and integrated star formation history of the Universe.
\end{abstract}

Keywords: GRB host galaxies - Infrared - Luminous Infrared Galaxies - Observational Cosmology PACS: 98.54.Ep - 98.70.Lt - 98.70.Rz - 98.80.Es

\section{INTRODUCTION}

In the past few years, the connection between long Gamma-Ray Bursts (hereafter GRBs) and the activity of massive star formation in distant galaxies has been established in a robust way [e.g., 1, 2, 3, 4]. Furthermore, GRBs are very little affected by dust extinction and they are likely detectable up to very high redshift. They could thus be used as probes of the whole star formation history of the Universe independently of all the usual biases affecting the current deep surveys. This statement is however based on the assumption that the production rate of GRBs as a function of redshift is strictly proportional to the amount of massive stars which are formed, with no redshift evolution of the parameters that may influence the trigger of these catastrophic events. In this context, the GRB host galaxies in a given redshift bin should be representative of the sources responsible for the bulk of the star-forming activity at this redshift. Comparing the properties of the GRB hosts with those of field sources is thus one way to test how well GRBs can signpost the sites of massive star formation in the distant Universe. 


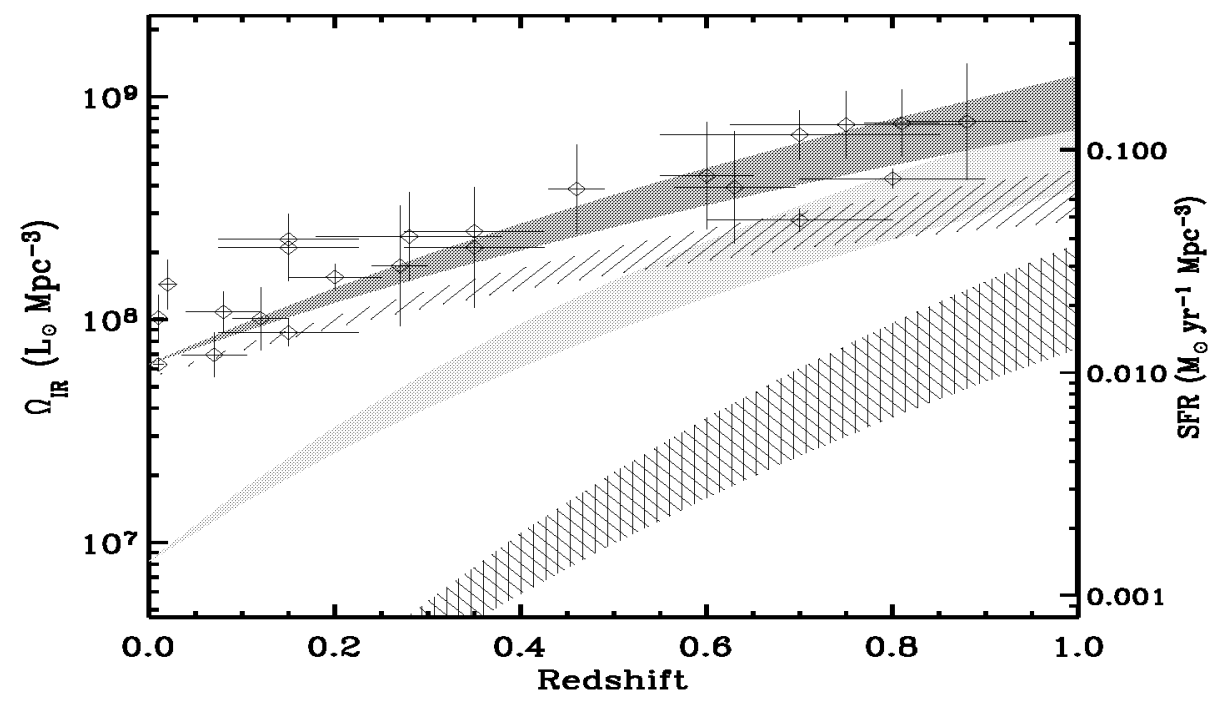

FIGURE 1. Evolution of the comoving IR energy density up to $z=1$ (dark shaded region) and the respective contributions from low luminosity galaxies (i.e., $\mathrm{L}_{\mathrm{IR}}<10^{11} \mathrm{~L} \odot$, lined area), "infrared luminous" sources (i.e., $\mathrm{L}_{\mathrm{IR}} \geq 10^{11} \mathrm{~L}_{\odot}$, light shaded region) and ULIRGs (i.e., $\mathrm{L}_{\mathrm{IR}} \geq 10^{12} \mathrm{~L}_{\odot}$, cross-hatched region). At $z \sim 1$ IR luminous galaxies represent $70 \pm 15 \%$ of the comoving IR energy density and dominate the star formation activity. From Le Floc'h et al. [7].

From the multi-wavelength deep surveys that were carried out in the last decade we know that the activity of star formation has been progressively shifting from very massive and very luminous starbursts at redshifts $z \sim 2-3$ from low-mass sub-luminous star-forming dwarves in the local Universe [e.g., 5, 6]. This is often called the "downsizing" of the cosmic evolution and it is illustrated in Fig. 1 which reports on the star formation history (SFH) of the Universe from $z=0$ to $z=1$. In this figure the integrated SFH has been decomposed into the contribution of galaxies classified as a function of their infrared (IR) luminosity (hence as a function of their star formation rate). We see that beyond $z \sim 0.7$, the so-called Luminous Infrared Galaxies and Ultra-Luminous InfraRed Galaxies (respectively LIRGs: $10^{11} \mathrm{~L}_{\odot} \leq \mathrm{L}_{\mathrm{IR}}=\mathrm{L}[8-1000 \mu \mathrm{m}] \leq 10^{12} \mathrm{~L}_{\odot}$, and ULIRGs: $\mathrm{L}_{\mathrm{IR}} \geq 10^{12} \mathrm{~L}_{\odot}$ ) dominate the SFH [7]. These IR-luminous starbursts are also intermediate or high-mass objects. If the long GRBs are tracing the star formation history, a significant fraction of these cosmic explosions should thus be observed in luminous and massive sources.

The most efficient facility currently accessible to the astronomical community for tracking the properties of massive, dusty and luminous starbursts at high redshift is the Spitzer Space Telescope. Spitzer was launched in August 2003 as the final mission of the NASA Great Observatories Program. This satellite is an infrared cold telescope with an $80 \mathrm{~cm}$-diameter mirror, equipped with two imagers called IRAC and MIPS and one spectrometer called IRS. IRAC observes in broad-band filters centered at 3.6, 4.5, 5.8 
and $8.0 \mu \mathrm{m}$, MIPS images the sky at 24, 70 and $160 \mu \mathrm{m}$ and the IRS instrument obtains mid-IR spectra of sources between 5 and $35 \mu \mathrm{m}$. Spitzer is now providing spectacular results which allow us better insights into the physical processes of dust emission in Galactic star-forming regions and nearby galaxies. It is also capable to detect very high redshift galaxies that are completely invisible in the deepest optical data ever taken by the Hubble Space Telescope [e.g., 8]. It routinely obtains mid-IR spectra of the SCUBA submillimeter sources at $z \sim 2-3$ [e.g., 9] and it has recently resolved up to $\sim 90 \%$ of the infrared background thanks to the unprecedented sensitivity of the MIPS instrument [e.g., 10, 11].

We thus used Spitzer to study the properties of several GRB host galaxies. Here we report on our observations and we analyze our data in the goal of testing whether GRBs are detected in massive and luminous IR starbursts at high redshift.

\section{OBSERVATIONS}

We targeted a sample of 16 GRB host galaxies using Spitzer as part of the IRS GTO program (PI: J.Houck). Each object was imaged with IRAC at 4.5 and $8 \mu \mathrm{m}$ down to $3.5 \mu \mathrm{Jy}$ and $20 \mu \mathrm{Jy}(3 \sigma)$ respectively, as well as with MIPS at $24 \mu \mathrm{m}$ down to $85 \mu \mathrm{Jy}(3 \sigma)$. These sensitivity limits are slightly shallower than those typically reached in the GTO surveys undertaken by Spitzer (e.g., $5 \sigma \sim 80 \mu$ Jy, [7]) but still reasonably deep. Assuming a typical conversion between the star formation rate (SFR) and the IR continuum emission (e.g., [12]), we infer that our $24 \mu \mathrm{m}$ data are sensitive to $\mathrm{SFR} \gtrsim 15 \mathrm{M}_{\odot} \mathrm{yr}^{-1}$ at $z \sim 1$ and $\mathrm{SFR} \gtrsim 150 \mathrm{M}_{\odot} \mathrm{yr}^{-1}$ at $z \sim 2$.

Our sample is composed of the host galaxies of all GRBs that were localized with a sub-arsecond accuracy between 1997 and July 1999, with the exception of the host of GRB 970228 that was replaced with the host of GRB 010222. A persistent submillimeter emission was in fact detected at the location of this burst [13], which makes it an obviously interesting target for IR observations. There was no other pre-selection using an a priori knowledge of e.g., redshifts, optical or near-infrared magnitudes, detections at other long wavelengths...

\section{RESULTS}

Most of the sources from our sample (i.e., $\sim 80 \%$ ) could not be detected with Spitzer (with neither IRAC nor MIPS), including the hosts of GRB 970508, GRB 980703 and GRB 010222 that have been proposed as potential ultra-luminous IR galaxies by [14], [15] and [13] respectively. One noticeable exception is the host of GRB 980613 presented as a merger-induced starburst by [16]. As seen in Fig. 2 this host galaxy is composed of several interacting knots. Two of them are clearly detected at $4.5 \mu \mathrm{m}$ with IRAC, and there is also evidence for a detection at 8.0 and $24 \mu \mathrm{m}$. These two components have very red $R-K$ colors, likely pointing to a dust-obscured starburst. Nonetheless, we note that the afterglow of the GRB was identified in another region located 2" away to the North, and which is not detected by Spitzer. This is therefore an interesting 


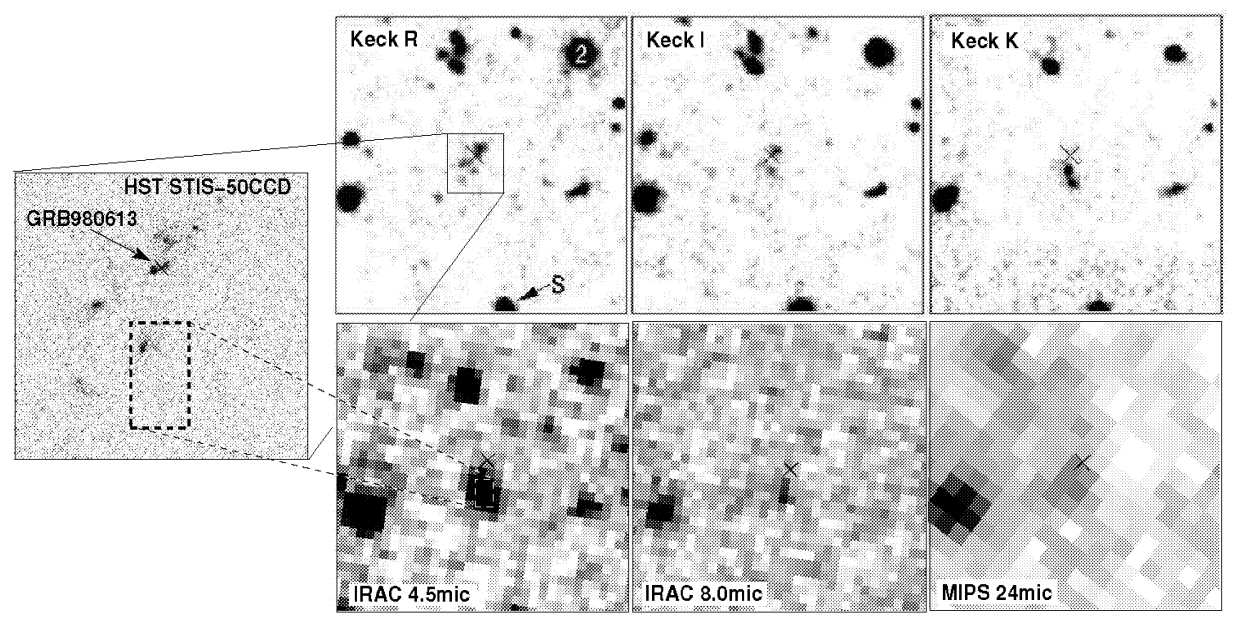

FIGURE 2. An optical and infrared view of the host of GRB 980613, an interacting system at $z=1.1$. In each panel the position of the GRB is indicated with a cross. This burst did not occur within the most IR-luminous (thus the most starbursting) component of the merger. This may reveal the influence of other physical parameters favoring the formation of such events (metallicity, efficiency of the star-forming activity, IMF, ...). From Djorgovski et al. [16], Holland et al. [17] and Le Floc'h et al. (submitted).

example of a GRB that did not occur in the most active star-forming environment of its host (see also [18]).

In summary, our sample of GRB host galaxies observed with Spitzer is not consistent with a population of massive and dusty luminous starbursts. This actually confirms previous optical and near-IR studies of the GRB hosts, performed either on a case-bycase basis (e.g. [1, 19]) or following a more statistical approach (e.g., [2, 20, 21, 22], and that led to the conclusion that these objects are mostly blue, sub-luminous, lowmass and young galaxies with a modest amount of star formation and a low level of dust extinction.

\section{IMPLICATIONS}

At $24 \mu \mathrm{m}$, the differential source number counts derived from the Spitzer deep surveys present a turn-over between 200 and $300 \mu \mathrm{Jy}$ when they are normalized to the Euclidean slope (e.g., [10]). This reveals that most of the IR background is produced by galaxies that are much brighter than the $3 \sigma$ sensitivity limit of our GRB host observations with MIPS. Using phenomenological models of IR galaxy evolution (e.g., [23]) we infered that more than $50 \%$ of our sample should have easily been detected by MIPS if the GRBs effectively trace the whole activity of star formation at high redshift. Our large fraction of non-detections at $24 \mu \mathrm{m}$ thus implies either that our sample is strongly biased or that the long GRBs preferentially occur in faint objects.

There is clearly one selection effect that may potentially affect our current sample. In fact these objects were identified mostly using the sub-arcsec location of optically-bright 
GRB afterglow transients. This type of selection may thus induce a bias against dusty sources. In favor of this interpretation, a fraction of the so-called "dark bursts" (i.e., GRBs with no detectable afterglows despite rapid and deep optical follow-ups) appears to be trully enshrouded behind dusty material. An example of these dusty dark GRBs is the GRB 970828, which was localized thanks to its radio afterglow [24]. Its host galaxy is actually one of the very few sources that were detected in our $24 \mu \mathrm{m}$ data, which clearly supports the scenario of a burst accompanied by an afterglow extinguished by dust. On the other hand, another large fraction of dark GRBs is also due to intrinsically faint bursts and GRBs with steep time decays. Furthermore it has been suggested that dust grains can be destroyed by hard X-ray emission along the line of sight of GRBs, which may substantially reduce the effect of extinction in the selection of star-forming environments with GRBs. Finally, we did not detect any IR emission toward the hosts of two other dark bursts (i.e., GRB 990506 and GRB 981226). This shows that these dark GRBs are not systematically associated with dusty star-forming galaxies, an interpretation already proposed by Barnard et al. [25] based on SCUBA observations of four dark GRB host galaxies. We infer that if this bias does exist it can not be important enough to explain all our non-detections by Spitzer.

Consequently, our results may reflect the influence of parameters more physically related to the environments where GRBs are produced, and which may explain why these GRBs preferentially take place in young, sub-luminous and blue objects rather than luminous and massive starbursts. For instance, the potential effect of a low metallicity in the GRB progenitor enveloppe is now intensively discussed by theorists (e.g, Woosley, MacFadyen et al., these proceedings) as it may clearly favor the trigger of such events. In fact, low metallicites have already been measured from the integrated spectra of several GRB hosts $[26,27]$, and this may also explain the statistically low luminosity of this GRB-selected population. Rotation effects and the implication of GRB progenitors within binary systems are also currently explored.

\section{SUMMARY}

As previously stated, our Spitzer observations reveal that long GRBs are statistically not observed in the massive and luminous infrared galaxies that dominate the activity of star formation in the early Universe. This actually confirms previous claims arguing for a population rather dominated by blue, young and low-mass objects, and it shows that the hosts of long GRBs are not representative of the sources that produced the bulk of stellar mass throughout the lifetime of the Universe. This tells us that the relation between massive star formation and long GRBs is likely much more complex than previously assumed, and it strongly suggests that the history of the GRB production rate can not be directly converted into the integrated star formation history. This disagreement might reflect the influence of specific parameters in the trigger of GRBs (e.g., metallicity, rotation effects, binarity, ....), that we need to understand if we want to control the use of GRBs as star formation tracers. 


\section{ACKNOWLEDGMENTS}

This conference in Washington DC was a really fruitful and pleasant meeting. The first author would like to thank all the people who contributed in making this event such a success.

\section{REFERENCES}

1. Fruchter, A. S., Thorsett, S. E., Metzger, M. R., et al. 1999, ApJ 519, L13

2. Sokolov, V. V., Fatkhullin, T. A., Castro-Tirado, A. J., et al. 2001, A\&A 372, 438

3. Bloom, J. S., Kulkarni, S. R., \& Djorgovski, S. G. 2002, AJ 123, 1111

4. Stanek, K. Z., Matheson, T., Garnavich, P. M., et al. 2003, ApJ 591, L17

5. Chary, R. \& Elbaz, D. 2001, ApJ 556, 562

6. Chapman, S. C., Blain, A. W., Ivison, R. J., \& Smail, I. R. 2003, Nature 422, 695

7. Le Floc'h, E., Papovich, C., Dole, H., et al. 2005, ApJ 632, 169

8. Mobasher, B., Dickinson, M., Fergusson, H., et al. 2005, ApJ in press (astro-ph/0509768)

9. Lutz, D., Valiante, E., Sturm, E., et al. 2005, ApJ 625, L83

10. Papovich, C., Dole, H., Egami, E., et al. 2004, ApJS 154, 70

11. Dole, H., Lagache, G., Puget, J.-L., et al. 2006 (submitted to A\&A)

12. Kennicutt, R. C. 1998, ARA\&A 36, 189

13. Frail, D. A., Bertoldi, F., Moriarty-Schieven, G. H., et al. 2002, ApJ 565, 829

14. Hanlon, L., Laureijs, R. J., Metcalfe, L., et al. 2000, A\&A 359, 941

15. Berger, E., Kulkarni, S. R., \& Frail, D. A. 2001, ApJ 560, 652

16. Djorgovski, S. G., Bloom, J. S., \& Kulkarni, S. R. 2003, ApJ 591, L13

17. Holland, S., Fynbo, J., Thomsen, B., et al. 2000, GRB Circular Network, 698

18. Hjorth, J., Thomsen, B., Nielsen, S. R., et al. 2002, ApJ 576, 113

19. Bloom, J. S., Odewahn, S. C., Djorgovski, S. G., et al. 1999, ApJ 518, L1

20. Le Floc'h, E., Duc, P.-A., Mirabel, I. F., et al. 2003, A\&A 400, 499

21. Courty, S., Björnsson, G., \& Gudmundsson, E. H. 2004, MNRAS 354, 581

22. Christensen, L., Hjorth, J., \& Gorosabel, J. 2004, A\&A 425, 913

23. Lagache, G., Dole, H., Puget, J.-L., et al. 2004, ApJS 154, 112

24. Djorgovski, S. G., Frail, D. A., Kulkarni, S. R., et al. 2001, ApJ 562, 654

25. Barnard, V. E., Blain, A. W., Tanvir, N. R., et al. 2003, MNRAS 338, 1

26. Prochaska, J. X., Bloom, J. S., Chen, H.-W., et al. 2004, ApJ 61 1, 200

27. Soderberg, A. M., Kulkarni, S. R., Berger, E., et al. 2004, ApJ 606, 994 